\title{
OVERTWISTED ENERGY-MINIMIZING CURL EIGENFIELDS
}

\author{
ROBERT GHRIST AND RAFAL KOMENDARCZYK
}

\begin{abstract}
This paper concerns topological and geometric properties of energy-minimizing solutions to the steady Euler equations for a fluid on a 3-dimensional manifold. Specifically, we consider energy-minimizing divergence-free eigenfields of the curl operator in dimension three from the perspective of contact topology. We give a negative answer to a question of Etnyre and the first author by constructing curl eigenfields which minimize $L^{2}$ energy on their co-adjoint orbit, yet are orthogonal to an OVERTWISTED contact structure. We then give progress toward the conjecture that $K$-contact structures on Seifert fibered manifolds always define TIGHT minimizers.
\end{abstract}

\section{INTRODUCTION}

Eigenfields of the curl operator form an important class of solutions to the steady Euler equations in dimension three. These equations model the velocity field of an inviscid, incompressible fluid flow on a Riemannian manifold [1]. It has been observed [7, 6, 9] that there is a correspondence between curl eigenfields and contact 1-forms in dimension three. Let $M$ be a 3-manifold and $\Omega^{1}(M)$ denote the 1-forms on $M$. Recall that a contact 1-form $\alpha \in \Omega^{1}(M)$ is one which satisfies $\alpha \wedge d \alpha \neq 0$. Such 1-forms have as their kernel a totally nonintegrable plane field known as a contact structure. The correspondence is this: any nonvanishing curl eigenfield is dual to a contact 1 -form via the Riemannian metric; and any contact 1 -form can be realized as dual to a nonvanishing curl eigenfield for some Riemannian structure.

This observation raises interesting questions concerning the interplay between fluid dynamical properties of curl eigenfields and topological properties of contact structures. More specifically, one can investigate how the topological tight/overtwisted dichotomy for contact structures relates to the physical properties of the fluid like energy, periodic orbits etc. In [9] (p.

1991 Mathematics Subject Classification. Primary: 58J05, 37J55; Secondary: 53D10.

Key words and phrases. eigenfield; contact structure; Euler equations; energy.

RG supported in part by NSF PECASE Grant \# DMS - 0337713.

RK supported in part by NSF Grant \# DMS - 0134408 . 
16) the authors asked if variational principles of the fluid flows descend to variational principles for the corresponding contact structures.

Question 1.1. Does a nonvanishing curl eigenfield which minimizes $\left(L^{2}\right)$ energy on its coadjoint orbit under the volume-preserving diffeomorphism group necessarily define a tight contact structure?

Using a recent result of the second author [12], we give a negative answer to this question. This involves constructing special $S^{1}$-invariant curl eigenfields on products $S^{1} \times \Sigma$, where $\Sigma$ is a closed orientable surface of genus $g>0$. In the second part of this note, we demonstrate that under additional symmetry conditions, the energy-minimization condition does yield a tightness constraint on the associated contact structures.

Throughout the paper, we use the language of differential forms and global analysis, as in [1]. We restrict attention to the class of volume-preserving vector fields and 1-forms exclusively.

\section{Contact Structures}

Let $(M, g)$ be a Riemannian 3-manifold. The CURL OPERATOR on 1-forms is $* d: \Omega^{1}(M) \rightarrow \Omega^{1}(M)$, where $*$ is the Hodge star operator and $d$ the exterior derivative. An EIGENFORM of curl is any 1-form $\alpha$ satisfying $* d \alpha=\mu \alpha$ for some $\mu \in \mathbb{R}$.

Given a nonvanishing curl eigenform $\alpha$ with nonzero eigenvalue $\mu \neq 0$, it easily follows that

$$
\alpha \wedge d \alpha=\alpha \wedge(\mu * \alpha) \neq 0
$$

Therefore, $\alpha$ is a CONTACT FORM and the plane field $\xi=\operatorname{ker}(\alpha)$ defines a contact structure - a nowhere integrable plane field on $M$. If $\alpha$ is the dual 1-form to a vector field, then $\xi$ is the orthogonal plane field. All contact structures in this paper are transversely orientable, as they are dual to globally-defined vector fields.

It has been known since the work of Bennequin and Eliashberg [2, 8] that there are two fundamentally different classes of contact structures.

Definition 2.1. A contact structure $\xi$ is OVERTWISTED if and only if there exists an embedded disk $D^{2} \subset M$ such that $D$ is transverse to $\xi$ near $\partial D$ but $\partial D$ is tangent to $\xi$. Any contact structure which is not overtwisted is called TIGHT.

As one might expect from the definition, it is rather difficult to determine if a given contact structure is tight or overtwisted. One of the more successful recent techniques for solving the classification problem involves examining the CHARACTERISTIC SURFACE. 
Definition 2.2. Let $X$ be a vector field preserving the contact plane distribution $\xi$, i.e., $\mathcal{L}_{X} \xi=0$. The characteristic surface $\Gamma_{X}$ is the set of tangencies of $X$ with $\xi$,

$$
\Gamma_{X}=\left\{p \in M: X_{p} \in \xi_{p}\right\}
$$

The following result is essential for our study of $S^{1}$-invariant curl eigenforms on circle bundles.

Theorem 2.3 (Giroux [10]). Let $\xi$ be an $S^{1}$-invariant contact structure on a principal circle bundle $\pi: P \rightarrow \Sigma$ over a closed oriented surface $\Sigma$. Let $\Gamma=$ $\pi\left(\Gamma_{S^{1}}\right)$ be a projection of the characteristic surface $\Gamma_{S^{1}}$ onto $\Sigma$. Denote by $e(P)$ be the Euler number of the bundle $P$. Then $\xi$ and all covers of $\xi$ are tight if and only if one of the following holds:

(i) For $\Sigma \neq S^{2}$ none of the connected components of $\Sigma / \Gamma$ is a disc.

(ii) For $\Sigma=S^{2}, e(P)<0$ and $\Gamma=\emptyset$.

(iii) For $\Sigma=S^{2}, e(P) \geq 0$ and $\Gamma$ is connected.

A contact structure all of whose covers are tight is called UNIVERSALLY TIGHT.

\section{ENERGY AND EIGENVALUES}

An important feature of any curl eigenform $\alpha$ is the fact that it extremizes the $L^{2}$-energy, defined as,

$$
E(\alpha)=\|\alpha\|_{L^{2}}^{2}=\int_{M} \alpha \wedge * \alpha,
$$

among all 1-forms obtained from $\alpha$ by pullbacks through volume preserving diffeomorphisms. The set of such forms, $\Xi_{\alpha}$, is the COADJOINT ORBIT of $\alpha$ under the action of the volume-preserving diffeomorphism group of $M$ :

$$
\Xi_{\alpha}=\left\{\beta: \beta=\varphi_{*}(\alpha), \varphi \in \operatorname{Diff}_{0}(M), \varphi_{*}(* 1)=* 1\right\}
$$

The question of energy minimization on the coadjoint orbit is more delicate, and closely related to spectral data. The following result is one of the few general results available:

[1]:

Proposition 3.1. A curl eigenform $\alpha_{1}$, (i.e. an eigenform of the curl operator $* d: \mathcal{H} \rightarrow \mathcal{H}, \mathcal{H}=\{\alpha: \delta \alpha=0\}=\{$ "divergence free" 1-forms $\}$ ). corresponding to the first eigenvalue $\mu_{1} \neq 0$ is a minimizer of the energy $E$ on $\Xi_{\alpha_{1}}$. 
Proof. The operator $* d$ is elliptic and consequently it's analytic realization is unbounded on $L_{\mathcal{H}}^{2}\left(M, \Lambda^{1} T^{*} M\right)$ (an $L^{2}$ - completion of $\mathcal{H}$, closed and selfadjoint; it has a compact inverse $* d^{-1}$ defined on the orthogonal complement of its kernel (see [15]). We can also choose an orthonormal basis of eigenforms $\left\{\alpha_{i}\right\}$ in $\left(L_{\mathcal{H}}^{2}\left(M, \Lambda^{1} T^{*} M\right),(\cdot, \cdot)_{L^{2}}\right)$ such that,

$$
* d^{-1} \alpha_{i}=\frac{1}{\mu_{i}} \alpha_{i}, \quad 0<\mu_{1}^{2} \leq \mu_{2}^{2} \leq \ldots \leq \mu_{i}^{2} \leq \ldots
$$

For an arbitrary $L^{2} 1$-form $\beta \in \operatorname{Im}(\delta)$ we have

$$
\left|\left(* d^{-1} \beta, \beta\right)_{L^{2}}\right|=\left|\sum_{i} \frac{1}{\mu_{i}}\left(\alpha_{i}, \beta\right)_{L^{2}}^{2}\right| \leq \frac{1}{\left|\mu_{1}\right|}(\beta, \beta)_{L^{2}}=\frac{1}{\left|\mu_{1}\right|} E(\beta) .
$$

One obtains a lower bound for the energy $E(\beta)$,

$$
E(\beta) \geq\left|\mu_{1}\right|\left|\left(* d^{-1} \beta, \beta\right)_{L^{2}}\right| .
$$

The above inequality becomes the equality if and only if $\beta$ is a $\mu_{1}$ - eigenform of $* d$. The claim follows from the fact that the HELICITY, $\left(* d^{-1} \beta, \beta\right)_{L^{2}}$, is invariant under volume preserving transformations see [1].

From now on we do not distinguish between operators defined on various spaces of smooth differential forms and their analytic realizations defined on $L^{2}$ - completions of those spaces.

The curl operator $* d: \mathcal{H} \rightarrow \mathcal{H}$ is a self-adjoint first-order elliptic operator, and the principal eigenvalue $\mu_{1}$ enjoys a variational characterization through the Rellich's quotient. Via Lemma 3.2 we have,

$$
\begin{gathered}
\mu_{1}=\inf _{\alpha \in \mathcal{H}_{0}^{\perp}} \frac{\left|(* d \alpha, \alpha)_{L^{2}}\right|}{\|\alpha\|_{L^{2}}^{2}} \Leftrightarrow \mu_{1}^{2}=\inf _{\alpha \in \mathcal{H}_{0}^{\perp}} \frac{\left(\Delta_{M}^{1} \alpha, \alpha\right)_{L^{2}}}{\|\alpha\|_{L^{2}}^{2}}, \\
\mathcal{H}_{0}^{\perp}=\operatorname{Ker}(* d)^{\perp}=\left\{\alpha \in \Omega^{1}(M): \alpha=\delta \beta, \text { for some } \beta \in \Omega^{2}(M)\right\},
\end{gathered}
$$

Observe that the curl squared is equal to the Hodge Laplacian , $(* d)^{2}=\delta d$, on $\mathcal{H}$. Therefore any curl eigenform $\alpha$, (i.e. $* d \alpha= \pm \mu \alpha$ ) is automatically a co-closed $\mu^{2}$-eigenform of the Hodge Laplacian i.e.

$$
\Delta_{M}^{1} \alpha=\delta d \alpha=* d * d \alpha=\mu^{2} \alpha,
$$

Clearly the curl $* d$ commutes with $\Delta_{M}^{1}$, therefore both of these operators are simultaneously diagonizable on $\mathcal{H}$ in a suitable orhonormal basis of curl eigenforms;

$\mathcal{H}=\bigoplus_{i=1}^{\infty} E^{\Delta}\left(\mu_{i}^{2}\right), \quad E^{\Delta}\left(\mu_{i}^{2}\right) \perp E^{\Delta}\left(\mu_{j}^{2}\right), i \neq j \quad 0<\mu_{1}^{2} \leq \mu_{2}^{2} \leq \ldots \leq \mu_{i}^{2} \leq \ldots$

where $E^{\Delta}\left(\mu_{i}^{2}\right)$ stands for the $\mu_{i}^{2}$-eigenspace of $\Delta_{M}^{1}$, and

$$
E^{\Delta}\left(\mu_{i}^{2}\right)=E^{* d}\left(\mu_{i}\right) \oplus E^{* d}\left(-\mu_{i}\right) .
$$


(We allow one of $E^{* d}\left(\mu_{i}\right), E^{* d}\left(-\mu_{i}\right)$ to be trivial.) We may conclude further that there exist two positive operators $\sqrt{\Delta}_{+}, \sqrt{\Delta}_{-}$, such that $* d={\sqrt{\Delta_{+}}}_{-}$ $\sqrt{\Delta}_{-}$.

The following useful fact, which can be traced back to work in [5], tells us how to effectively find a basis of curl eigenforms from a basis of co-closed $\Delta_{M}^{1}$-eigenforms.

Lemma 3.2. Any curl $\mu$-eigenform is automatically a co-closed $\mu^{2}$-eigenform of the Laplacian $\Delta_{M}^{1}$. Conversely, given a co-closed $\mu^{2}$-eigenform $\alpha \in \Omega^{1}(M)$ of $\Delta_{M}^{1}$ there exists a corresponding $\pm \mu$-curl eigenform $\beta_{ \pm} \in \Omega^{1}(M)$ given by

$$
\beta_{ \pm}=\mu \alpha \pm * d \alpha
$$

Proof. The first claim follows from (7). The second claim we verify by a direct calculation. Let $\beta_{ \pm}$be defined by (8), we will show: $* d \beta_{ \pm}= \pm \mu \beta_{ \pm}$. Since $* d * d=\delta d=\left.\Delta_{M}^{1}\right|_{\mathcal{H}}$, and $\delta \alpha=0$ we obtain

$$
* d \beta_{ \pm}=\mu * d \alpha \pm \Delta_{M}^{1} \alpha .
$$

Secondly $\Delta_{M}^{1} \alpha=\mu^{2} \alpha$, therefore

$$
* d \beta_{ \pm}=\mu * d \alpha \pm \mu^{2} \alpha= \pm \mu \beta_{ \pm} .
$$

\section{OVERTWISTED PRINCIPAL EIGENFIELDS}

For the purpose of producing an overtwisted principal curl eigenform we assume that $M$ is a trivial bundle $P=S^{1} \times \Sigma$ and the metric $g$ on $P$ is a product metric $g=1 \oplus g_{\Sigma}$ such that fibres are of constant length $l$. We will construct our example using a sequence of lemmas.

In the case of the product $(P, g), P=S^{1} \times \Sigma, g=1 \oplus g_{\Sigma}$, the space of smooth 1-forms $\Omega^{1}(P)$ decomposes with respect to the $L^{2}$-inner product induced by the metric $g$ as,

$$
\Omega^{1}(P)=\Omega_{N}^{1}(P) \oplus \Omega_{T}^{1}(P)
$$

where,

$$
\begin{gathered}
\Omega_{N}^{1}(P)=\left\{\alpha \in \Omega^{1}(P): \alpha=f \eta, f \in C^{\infty}(P)\right\}, \\
\Omega_{T}^{1}(P)=\Omega_{N}^{1}(P)^{\perp} \cap \Omega^{1}(P), \\
\Omega_{T}^{1}(P)=\left\{\alpha \in \Omega^{1}(P): \alpha\left(X_{\eta}\right)=0\right\},
\end{gathered}
$$

with $\eta$ and $X_{\eta}$ being the tangent 1-form and vector field (resp.) to the $S^{1}$ fibres of unit magnitude $\left(\left\|X_{\eta}\right\|_{g}=1\right)$. The following lemma is another consequence of the product metric assumption. 
Lemma 4.1. The Laplacian $\Delta_{P}^{1}$ preserves $\Omega_{N}^{1}(P), \Omega_{T}^{1}(P)$ and for $\alpha=f \eta+\beta$, f $\eta \in \Omega_{N}^{1}(P), \beta \in \Omega_{T}^{1}(P)$ we have the following formula for the Laplacian at a point $(t, q) \in S^{1} \times \Sigma$,

$$
\Delta_{P}^{1} \alpha=\left(-\mathcal{L}_{\eta}^{2} f+\Delta_{\Sigma}^{0} f_{t}\right) \eta+\left(-\mathcal{L}_{\eta}^{2} \beta+\Delta_{\Sigma}^{1} \beta_{t}\right), \quad \text { at } \quad(t, q),
$$

where $f_{t}=\left.f\right|_{\{t\} \times \Sigma} \in C^{\infty}(\Sigma)$ and $\Delta_{\Sigma}^{0}$ is the scalar Laplacian on $\Sigma$. Similarly $\beta_{t}=\left.\beta\right|_{\{t\} \times \Sigma}$ and $\Delta_{\Sigma}^{1}$ is the 1-form Laplacian on $\Sigma$.

Proof. The first claim follows immediately from the formula (10). We justify (10) by a direct calculation in the $X_{\eta}$ - invariant frame $\left\{X_{1}, X_{2}, X_{3}\right\}, X_{1}=$ $X_{\eta}$, (denote the co-frame by $\left\{\eta_{i}\right\}, \eta_{1}=\eta$ ) on $P$, where $X_{2}, X_{3}$ are tangent to the $\Sigma$ fibers. Denote: $\nabla_{i}=\nabla_{X_{i}}$ and recall the following formulas (see e.g. [11])

$$
\begin{aligned}
\nabla_{i} X_{j} & =\Gamma_{i j}^{k} X_{k}, \quad \nabla_{i} \eta_{k}=-\Gamma_{i j}^{k} \eta_{j}, \quad \Gamma_{i j}^{k}=-\Gamma_{i k}^{j}, \\
\nabla_{i i}^{2} & =-\nabla_{i} \nabla_{i}+\Gamma_{i i}^{j} \nabla_{j}, \quad \Delta^{0}=-\nabla_{i i}^{2} .
\end{aligned}
$$

The well known Weitzenblöck formula (see [11] p. 138) for the $k$-from Laplacian $\Delta^{k}$ tells us $(k=1)$ :

$$
\begin{aligned}
\Delta^{1} \alpha & \left.=-\nabla_{i i}^{2} \alpha-\eta_{i} \wedge\left(X_{j}\right\lrcorner R\left(X_{i}, X_{j}\right) \alpha\right), \quad \alpha \in \Omega^{1}(M), \\
R\left(X_{i}, X_{j}\right) \alpha & =\nabla_{i} \nabla_{j} \alpha-\nabla_{j} \nabla_{i} \alpha-\nabla_{\left[X_{i}, X_{j}\right]} \alpha .
\end{aligned}
$$

In the product metric we may choose locally an $X_{\eta}$ - invariant frame $\left\{X_{i}\right\}$, meaning:

$$
\left[X_{1}, X_{j}\right]=-\left[X_{j}, X_{1}\right]=0, \quad\left[X_{2}, X_{3}\right] \in T \Sigma .
$$

Consequently (by $\Gamma_{i j}^{k}=\frac{1}{2}\left\langle\left[X_{i}, X_{j}\right], X_{k}\right\rangle-\left\langle\left[X_{j}, X_{k}\right], X_{i}\right\rangle+\left\langle\left[X_{k}, X_{i}\right], X_{j}\right\rangle$ ):

$$
\begin{aligned}
\Gamma_{i j}^{k} & =0, \quad \text { if one of the indices } i, j, k=1, \\
\nabla_{1} \Gamma_{i j}^{k} & =0, \quad \text { for all } i, j, k \\
R\left(X_{i}, X_{j}\right) \eta_{r} & =0, \quad \text { if one of the indices } i, j, k, r=1,
\end{aligned}
$$

where (16) is a consequence of the following

$$
\left.X_{k}\right\lrcorner R\left(X_{i}, X_{j}\right) \eta_{r}=\nabla_{j} \Gamma_{i k}^{r}-\nabla_{i} \Gamma_{j k}^{r}+\Gamma_{j n}^{r} \Gamma_{i k}^{n}-\Gamma_{i n}^{r} \Gamma_{j k}^{n}+\left(\Gamma_{i j}^{n}-\Gamma_{j i}^{n}\right) \Gamma_{n k}^{r} .
$$

In turn we obtain,

$$
\nabla \eta_{1}=0, \quad \nabla_{1} \eta_{k}=0, \quad \nabla_{2} \eta_{i}=-\Gamma_{i 3}^{2} \eta_{3}, \quad \nabla_{3} \eta_{i}=-\Gamma_{i 2}^{3} \eta_{2} .
$$

Let $\alpha=f \eta+\beta$, then $\Delta_{P}^{1} \alpha=\Delta_{P}^{1}\left(f \eta_{1}\right)+\Delta_{P}^{1} \beta$, we obtain from (13), (16),

$$
\begin{aligned}
\Delta_{P}^{1}\left(f \eta_{1}\right) & =-\nabla_{i i}^{2}\left(f \eta_{1}\right)=-\nabla_{i} \nabla_{i}\left(f \eta_{1}\right)+\Gamma_{i i}^{j} \nabla_{j}\left(f \eta_{1}\right) \\
\text { (17) }: & =\left(-\nabla_{i} \nabla_{i} f+\Gamma_{i i}^{j} \nabla_{j} f\right) \eta_{1} \\
\text { (14) }- \text { (16) : } & =\left(-\nabla_{1} \nabla_{1} f-\nabla_{2} \nabla_{2} f+\Gamma_{22}^{3} \nabla_{3} f-\nabla_{3} \nabla_{3} f+\Gamma_{33}^{2} \nabla_{2} f\right) \eta_{1}
\end{aligned}
$$


Treating $f=f_{t}$ as a family of functions $f_{t} \in C^{\infty}(\Sigma)$ dependent on $t \in S^{1}$, by (12) we have,

$$
\begin{gathered}
-\nabla_{2} \nabla_{2} f_{t}+\Gamma_{22}^{3} \nabla_{3} f_{t}-\nabla_{3} \nabla_{3} f_{t}+\Gamma_{33}^{2} \nabla_{2} f_{t}=\Delta_{\Sigma}^{0} f_{t}, \quad \text { therefore } \\
\Delta_{P}^{1}(f \eta)=\left(-\nabla_{1} \nabla_{1} f+\Delta_{\Sigma}^{0} f_{t}\right) \eta=\left(-\mathcal{L}_{\eta}^{2} f+\Delta_{\Sigma}^{0} f_{t}\right) \eta .
\end{gathered}
$$

Similar reasoning applies to $\beta=a_{2} \eta_{2}+a_{3} \eta_{3}$, by (17) and (16) we have

$$
\begin{aligned}
\left.\left(-\nabla_{1} \nabla_{1}+\Gamma_{11}^{j} \nabla_{j}\right) \beta-\eta_{1} \wedge\left(X_{j}\right\lrcorner R\left(X_{1}, X_{j}\right) \beta\right) & =-\nabla_{1} \nabla_{1}\left(a_{2} \eta_{2}+a_{3} \eta_{3}\right) \\
= & -\left(\nabla_{1} \nabla_{1} a_{2}\right) \eta_{2}-\left(\nabla_{1} \nabla_{1} a_{3}\right) \eta_{3}=-\mathcal{L}_{\eta}^{2} \beta
\end{aligned}
$$

Treating $\beta=\beta_{t}$ as a family of 1 -forms $\beta_{t} \in \Omega^{1}(\Sigma)$, and using (17), (16) and (13) one shows,

$$
\left.\sum_{i=2}^{3}\left\{-\nabla_{i} \nabla_{i} \beta+\Gamma_{i i}^{j} \nabla_{j}\right) \beta-\eta_{i} \wedge\left(X_{j}\right\lrcorner R\left(X_{i}, X_{j} \beta\right)\right\}=\Delta_{\Sigma}^{1} \beta_{t} .
$$

Lemma 4.2. On the product manifold $P=S^{1} \times \Sigma, g=1 \oplus g_{\Sigma}$ with constant length l fibres. The first eigenvalue $\mu_{1}$ of the curl operator satisfies,

$$
\mu_{1}^{2}=\min \left\{\nu_{1},\left(\frac{2 \pi}{l}\right)^{2}\right\}, \quad \text { where } \quad \nu_{1}=\inf _{f \in L^{2}(\Sigma), f \neq c o n s t}\left\{\frac{\left(\Delta_{\Sigma}^{0} f, f\right)_{L^{2}}}{\|f\|_{L^{2}}^{2}}\right\} .
$$

Proof. From the decomposition (9) and the fact that $\Delta_{P}^{1}$ preserves $\Omega_{T}^{1}(P)$ and $\Omega_{N}^{1}(P)$ (see Lemma4.1) we have

$$
\mu_{1}^{2}=\min \left\{\mu_{1, T}^{2}, \mu_{1, N}^{2}\right\} ; \quad \mu_{1, r}^{2}=\inf _{\alpha \in \mathcal{H}_{0}^{\perp} \cap \Omega_{r}^{1}(P)}\left\{\frac{\left(\Delta_{P}^{1} \alpha, \alpha\right)_{L^{2}}}{\|\alpha\|_{L^{2}}^{2}}\right\} r=T, N .
$$

In order to calculate $\mu_{1, N}^{2}$ notice that for any $\alpha \in \mathcal{H} \cap \Omega_{N}^{1}(P), \alpha=f \eta$, the function $f$ is constant on the fibres; hence $f \in C^{\infty}(\Sigma)$. Indeed, $\delta \alpha=0$, and, since $\nabla \eta=0$ in the adapted frame $\left\{X_{1}, X_{2}, X_{3}\right\}$ with $X_{1}=X_{\eta}$, we obtain

$$
\left.\left.0=\delta \alpha=X_{i}\right\lrcorner \nabla_{i} \alpha=X_{i}\right\lrcorner\left(\nabla_{i} f \eta+f \nabla_{i} \eta\right)=\nabla_{1} f=X_{\eta} f .
$$

From (10) we conclude that for any $\alpha=f \eta \in \mathcal{H} \cap \Omega_{N}^{1}(P)$, we have: $\Delta_{\Sigma}^{1} \alpha=$ $\left(\Delta_{\Sigma}^{0} f\right) \eta$ and consequently,

$$
\begin{aligned}
& \left(\Delta_{P}^{1} \alpha, \alpha\right)_{L^{2}}=\left(\Delta_{\Sigma}^{0} f \eta, f \eta\right)_{L^{2}}=\int_{S^{1} \times \Sigma}\left(f \Delta_{\Sigma}^{0} f\right) \eta \wedge * \eta \\
& \quad=\int_{S^{1}} \int_{\Sigma} f \Delta_{\Sigma}^{0} f=l \int_{\Sigma} f \Delta_{\Sigma}^{0} f=l\left(\Delta_{\Sigma}^{0} f, f\right)_{L^{2}} \\
& \text { and } \quad\|\alpha\|_{L^{2}}^{2}=(\alpha, \alpha)_{L^{2}}=\int_{S^{1} \times \Sigma} f^{2} \eta \wedge * \eta=l\|f\|_{L^{2}}^{2}
\end{aligned}
$$


where $\eta \wedge * \eta=* 1$. As a result, (6),

$$
\mu_{1, N}^{2}=\nu_{1}, \quad \nu_{1}=\inf _{f \in L^{2}(\Sigma), f \neq \text { const }}\left\{\frac{\left(\Delta_{\Sigma}^{0} f, f\right)_{L^{2}}}{\|f\|_{L^{2}}^{2}}\right\} .
$$

In other words $\mu_{1, N}^{2}$ is equal to the first eigenvalue of the scalar Laplacian $\Delta_{\Sigma}^{0}$ on $\Sigma$.

In order to calculate $\mu_{1, T}^{2}$ we first calculate the orthogonal basis of eigenforms of $\mathcal{H} \cap \Omega_{T}^{1}(P)$. Let $\left\{\beta_{m}\right\}$ be an orthonormal basis of $\Delta_{\Sigma}^{1}$ - eigenforms on $\operatorname{Ker}\left(\Delta_{\Sigma}^{1}\right) \oplus \operatorname{Im}(\delta) \subset L^{2}\left(\Lambda^{1} T^{*} \Sigma\right)$, define for all $m, n \in \mathbb{Z}^{+}$:

$$
\begin{gathered}
h_{0}=g_{0}=1, \quad h_{n}=\cos \left(\frac{2 \pi n t}{l}\right), g_{n}=\sin \left(\frac{2 \pi n t}{l}\right), \\
\alpha_{n m}^{g}=g_{n} \beta_{m}, \quad \text { and } \quad \alpha_{n m}^{h}=h_{n} \beta_{m} .
\end{gathered}
$$

Clearly $\left\{\alpha_{n m}^{g}, \alpha_{n m}^{h}\right\}$ is a set of 1-eigenforms of $\Delta_{P}^{1}$ on $\mathcal{H} \cap \Omega_{T}^{1}(P)$. Indeed, from (10) we have,

$$
\Delta_{P}^{1} \alpha_{m n}^{r}=\gamma_{m n}^{r} \alpha_{m n}^{r}, \quad \gamma_{m n}^{r}=\left(\frac{2 \pi n}{l}\right)^{2}+\tilde{\nu}_{m}
$$

where $\tilde{\nu}_{m}$ is the $m$-th eigenvalue of $\Delta_{\Sigma}^{1}$. One easily shows that $\left\{\alpha_{n m}^{g}, \alpha_{n m}^{h}\right\}$ is an orthonormal basis of $\mathcal{H} \cap \Omega_{T}^{1}(P)$. Consequently, all eigenforms of $\Delta_{P}^{1}$ on $\mathcal{H} \cap \Omega_{T}^{1}(P)$ are listed in (20), and we have

$$
\mu_{1, T}^{2}=\min \left\{\left(\frac{2 \pi}{l}\right)^{2}, \tilde{\nu}_{1}\right\}
$$

It is left to show that $\tilde{\nu}_{1}=\nu_{1}$. By the Hodge decomposition theorem:

$$
\Omega^{1}(\Sigma)=\operatorname{Ker}\left(\Delta_{\Sigma}^{1}\right) \oplus \operatorname{Im}\left(d_{\Sigma}\right) \oplus \operatorname{Im}\left(\delta_{\Sigma}\right) .
$$

Moreover, $\Omega^{0}(\Sigma) \simeq \Omega^{2}(\Sigma)$ through the Hodge-star isometry. We conclude that $\operatorname{Im}\left(\delta_{\Sigma}\right)=\left\{*_{\Sigma} d f ; f \in C^{\infty}(\Sigma)\right\}$. Since $\Delta_{\Sigma}^{1}$ commutes with $*_{\Sigma} d$, any $\nu_{m}$-eigenfunction $f_{m}$ results in a $\nu_{m}$-eigenform $*_{\Sigma} d f_{m}$. Therefore $\tilde{\nu}_{1}=\nu_{1}$ and the lemma follows from (18), (19), and (21).

In [12] the following theorem was proved:

Theorem 4.3 ([12]). For an arbitrary closed compact orientable surface $\Sigma$, there exists a smooth metric $g_{\Sigma}$ such that a nodal set $f_{1}^{-1}(0)$ of the principal eigenfunction $f_{1}$ of $\Delta_{\Sigma}^{0}$ is a single embedded circle which bounds a disc in $\Sigma$.

Combining the above theorem with Lemma 4.2 results in the following,

Theorem 4.4. Let $\Sigma \neq S^{2}$ be an orientable surface of an arbitrary nonzero genus. One can prescribe a metric $g_{\Sigma}$ on $\Sigma$ such that there exist an overtwisted curl eigenfield $v$ on the product manifold $\left(S^{1} \times \Sigma, 1 \oplus g_{\Sigma}\right)$ which minimizes the energy (2) on the coadjoint orbit $\Xi_{\alpha}$. 
Proof. In the first step we choose a metric $g_{\Sigma}$ on $\Sigma \neq S^{2}$ constructed in Theorem 4.3 and assume that the length of fibres in $\left(S^{1} \times \Sigma, 1 \oplus g_{\Sigma}\right)$ is equal to $l$. By Lemma4.2 we may choose $l$ small so that the first eigenvalue satisfies $\mu_{1}=\nu_{1}$. The proof of 4.2 implies that the corresponding eigenspace $E^{\Delta}\left(\mu_{1}^{2}\right)$ is spanned by two independent co-closed $\mu_{1}^{2}$ - eigenforms of $\Delta_{P}^{1}: \alpha_{1}=f_{1} \eta$, and $\alpha_{2}=* d_{\Sigma} f_{1}$. (The dimension is two since $g_{\Sigma}$ can be chosen in a residual subset of an open set of metrics: see, [12].) By earlier considerations $E^{\Delta}\left(\mu_{1}^{2}\right)=E^{* d}\left(\mu_{1}\right) \oplus E^{* d}\left(-\mu_{1}\right)$ and $E^{\Delta}\left(\mu_{1}^{2}\right)$ is spanned by two independent $\pm \mu_{1}$ - curl eigenforms. Choosing any linear combination of $\alpha_{1}$ and $\alpha_{2}$ Lemma 3.2 leads to $\pm \mu_{1}$ - curl eigenforms given by,

$$
\beta_{ \pm}=f_{1} \eta \pm *_{\Sigma} d f_{1} \text {. }
$$

These forms are nonvanishing since the set of zeros is clearly equal to the singular part of the nodal set of $f_{1}$. This singular part is empty for a generic choice of metric (see [14]). Both forms are $S^{1}$-invariant and overtwisted by Theorem 2.3. Indeed, the projection of the characteristic surface $\Gamma_{S^{1}}$ of $\alpha_{ \pm}$ onto $\Sigma$ is equal to $f_{1}^{-1}(0)$, the nodal set of $f_{1}$. By the choice of the metric $\pi\left(\Gamma_{S^{1}}\right)$ bounds a disk. Now, the dual curl eigenfields $\beta_{ \pm}^{\#}$ minimize energy (2) on $\Xi_{\beta_{ \pm}}$due to Proposition 3.1 .

Remark 4.5. By perturbing a product metric on $P$ the eigenvalues $\pm \mu_{1}$, "split apart" giving only one of minimal absolute eigenvalue, and one dimensional eigenspaces. If the perturbation is small then the resulting eigenform will be $C^{0}$ - close to $\beta_{+}$( or $\beta_{-}$), and define an isotopic contact structure (by Grey's Theorem, [8]), which in turn must be an overtwisted minimizer.

\section{SYMMETRY AND TIGHT ENERGY MINIMA}

There are certain cases for which principal curl eigenforms can never be overtwisted. Courant's theorem on nodal sets quickly yields such a rigidity result for $S^{1} \times S^{2}$ :

Proposition 5.1. Let $M=S^{1} \times S^{2}$ with any product metric giving the $S^{1}$ fibres a constant length $l$. Then the principal eigenform of curl on $M$ is never overtwisted.

Proof. It follows from Lemma 4.2 that if the first eigenvalue $\mu_{1}$ is equal to $\frac{2 \pi}{l}$ then the curl eigenforms have zeros (since the dual vector fields are tangent to $S^{2}$ ). If $\mu_{1}=\nu_{1}$ Theorem 2.3 tells us that a contact form is tight if and only if the projection of $\Gamma_{S^{1}}=f_{1}^{-1}(0)$ is a single circle. On the other hand Courant's theorem implies that the principal eigenfunction on a closed surface always has exactly two nodal domains, which in turn implies tightness. 
For the more general case, tightness can be forced by additional symmetry. Let $\left(M, g_{M}\right)$ be a Riemannian 3- manifold which admits a unit Killing vector field $X$ orthogonal to a contact structure $\xi=X^{\perp}$. Choosing a local frame of vector fields $\left\{X_{1}, X_{2}, X_{3}\right\}, X_{1}=X$, since $\mathcal{L}_{X} g_{P}=0$ and consequently: $g_{P}\left(\nabla_{V} X, W\right)=-g_{P}\left(V, \nabla_{W} X\right)$, we obtain

$$
\begin{gathered}
\Gamma_{11}^{2}=\Gamma_{11}^{3}=\Gamma_{21}^{2}=\Gamma_{31}^{3}=0, \quad \Gamma_{i j}^{k}=-\Gamma_{i k}^{j}, \\
\Gamma_{31}^{2}=\Gamma_{21}^{3}=\lambda,
\end{gathered}
$$

where $\nabla_{i} X_{j}=\Gamma_{i j}^{k} X_{k}$, and $\lambda$ is an invariant independent on the choice of $\left\{X_{2}, X_{3}\right\}$. Cartan's equations of structure imply that dual 1-form $\eta=X^{b}$ satisfies

$$
* d \eta=2 \lambda \eta, \quad \lambda \in C^{\infty}(P) .
$$

In the case $\lambda(x)=\lambda=$ const, the triple $\left(M, \eta, g_{M}\right)$ is called $(K, \lambda)$ - STRUCTURE on $M$ ([13]), in which case $\eta$ defines a curl eigenform on $M$. The $(K, 1)$ - manifolds are also known as $K$-CONTACT manifolds or equivalently, in dimension 3, Sasakian manifolds, $([4,13])$, the Hopf fields on the round $S^{3}$ are the classical example (see [1]). We remark here that if $M$ admits a $(K, \lambda)$ - structure then it is topologically a Seifert manifold (see [13] for the proof).

Now we focus on the special case of $\left(M, \eta, g_{M}\right)$, namely the case of a principal $S^{1}$-bundle over a closed orientable surface $\Sigma$, equipped with a bundle metric $g_{P}$. All fibres are geodesics, as the bundle metric is invariant under the action of a unit Killing field $X$ tangent to the fibres, and the projection $\pi: P \rightarrow \Sigma$ is a Riemannian submersion.

By Theorem $2.3 \eta$ is necessarily tight since the contact plane distribution is orthogonal to the fibres.

Denote by $\mathcal{H}_{S^{1}}$ the subspace of $S^{1}$ - invariant 1 -forms in $\mathcal{H} \subset \Omega^{1}(P)$.

Proposition 5.2. Any curl eigenform $\eta$ defined by a $(K, \lambda)$-structure on $\left(P, g_{P}\right)$ is always energy-minimizing on $\mathcal{H}_{S^{1}} \cap \Xi_{\eta}$. Let $\nu$ be the first nonzero eigenvalue of the scalar Laplacian $\Delta_{\Sigma}^{0}$ on $\Sigma$. If $\nu>3 \lambda^{2}$ then $\eta$ is a principal curl eigenform on $\mathcal{H}_{S^{1}}$.

Proof. We provide the proof of the first claim for $\lambda>0$ (in the case of $\lambda<0$ the reasoning is analogous). The space $\mathcal{H}_{S^{1}}$ decomposes as

$$
\mathcal{H}_{S^{1}}=\mathcal{H}_{S^{1}}^{+} \oplus \mathcal{H}_{S^{1}}^{-},
$$

where $\mathcal{H}_{S^{1}}^{ \pm}$is a subspace spanned by positive/negative curl eigenforms. We need to show that $\eta$ is an energy minimizer on $\mathcal{H}_{S^{1}} \cap \Xi_{\eta}$. Given a volume preserving diffeomorphism $\varphi: P \rightarrow P$ we denote $\eta_{\varphi}=\varphi_{*}(\eta) \in \Xi_{\eta}$. Under the assumptions on the $\varphi$ action, $\eta_{\varphi} \in \mathcal{H}_{S^{1}} \cap \Xi_{\eta}$. We expand $\eta_{\varphi}$ in the 
eigenbasis of curl eigenforms (4), $\eta_{\varphi}=\sum_{i>0} c_{i}^{+} \alpha_{i}^{+}+\sum_{i<0} c_{i}^{-} \alpha_{i}^{-}$, where $\left\{\alpha_{i}^{ \pm}\right\}$span $\mathcal{H}_{S^{1}}$. Since the helicity $\left(* d^{-1} \eta_{\varphi}, \eta_{\varphi}\right)$ is invariant under $\varphi$, as in (5), we obtain

$$
0<\frac{E(\eta)}{2 \lambda}=\left(* d^{-1} \eta, \eta\right)=\left(* d^{-1} \eta_{\varphi}, \eta_{\varphi}\right)=\sum_{i \geq 0} \frac{\left(c^{+}\right)^{2}}{\mu_{i}^{+}}+\sum_{i<0} \frac{\left(c^{-}\right)^{2}}{\mu_{i}^{-}}
$$

where $\mu_{i}^{ \pm}$, positive/negative eigenvalues of $* d$ on $\mathcal{H}_{S^{1}}$. Since the second sum is negative we can estimate $\mu_{1}^{+}\left(* d^{-1} \eta, \eta\right) \leq E\left(\eta_{\varphi}\right)$. To finish the proof it suffices to show that $2 \lambda=\mu_{1}^{+}$, then we obtain $E(\eta) \leq E\left(\eta_{\varphi}\right)$ which proves the claim.

We derive the equality $2 \lambda=\mu_{1}^{+}$by a calculation in a local (co)frame $\left\{X_{i}\right\}\left(\left\{\eta_{i}\right\}\right)$, $X_{1}=X$, where $X$ is the Killing field tangent to the fibres and ker $\alpha_{1}=$ $\operatorname{span}\left\{X_{2}, X_{3}\right\}$. We adapt the notation from Lemma 3.2. Let $\alpha_{1}=a_{i} \eta^{i}=$ $f \eta+\beta$ be the curl eigenform satisfying

$$
* d \alpha_{1}=\mu_{1}^{+} \alpha_{1} .
$$

Since, $\mathcal{L}_{X} \alpha_{1}=0$, using the Cartan formula we obtain:

$$
\begin{aligned}
0 & \left.=\mathcal{L}_{X} \alpha=\mu_{1}^{+} X\right\lrcorner * \alpha+d f \\
-d f=-\nabla_{i} f \eta^{i} & \left.=\mu_{1}^{+} X_{1}\right\lrcorner \alpha \\
d f=\nabla_{1} f \eta^{1}+\nabla_{2} f \eta^{2}+\nabla_{3} f \eta^{3} & =\mu_{1}^{+}\left(a_{2} \eta^{3}-a_{3} \eta^{2}\right) .
\end{aligned}
$$

This leads to the following equations,

$$
\nabla_{1} f=0, \quad \nabla_{2} f=-\mu_{1}^{+} a_{3}, \quad \nabla_{3} f=\mu_{1}^{+} a_{2} .
$$

Applying (25) and (26) to $d \alpha=\nabla_{i} a_{k} \eta^{i} \wedge \eta^{k}-a_{k} \Gamma_{j i}^{k} \eta^{i} \wedge \eta^{j}$ yields

$$
\mu_{1}^{+} f=\frac{1}{\mu_{1}^{+}}\left(-\nabla_{2} \nabla_{2} f-\nabla_{3} \nabla_{3} f+\Gamma_{33}^{2} \nabla_{2} f+\Gamma_{22}^{3} \nabla_{3} f\right)+2 \lambda f .
$$

By (12) and (22), (23) we obtain the following equation for $f$ :

$$
\Delta_{P}^{0} f=\mu_{1}^{+}\left(\mu_{1}^{+}-2 \lambda\right) f .
$$

Equations (26) imply that for $\alpha_{1}$ to be nontrivial, $f$ cannot be a constant zero function. Since $\Delta_{P}^{0}$ is a positive operator we conclude that $\mu_{1}^{+} \geq 2 \lambda$; consequently, $\mu_{1}^{+}=2 \lambda$ because $\mu_{1}^{+}$is the first positive eigenvalue.

Equations (27) and (28) are valid for any $S^{1}$-invariant $\mu$-eigenform. Because $\pi$ is the Riemannian submersion: $\Delta_{P}^{0}(h \circ \pi)=\pi \circ \Delta_{\Sigma}^{0} h, h \in C^{\infty}(\Sigma)$, and the proof of the second statement follows from the equation: $\nu=\mu(\mu-2 \lambda)$. Indeed, for $\gamma^{2}=\nu, \gamma>0$, we obtain

$$
\mu^{2}-2 \lambda \mu+\gamma^{2}=0, \quad \bar{\mu}=\lambda+\sqrt{\lambda^{2}+\gamma^{2}}, \underline{\mu}=\lambda-\sqrt{\lambda^{2}+\gamma^{2}},
$$


where $\bar{\mu}, \underline{\mu}$ are the roots of the equation. Consequently,

$$
\begin{gathered}
\mu_{1}^{-} \in(-\infty,-\gamma), \text { if } \quad \mu_{1}^{+}=\lambda>0 \\
\mu_{1}^{+} \in(\gamma,+\infty), \text { if } \quad \mu_{1}^{-}=\lambda<0,
\end{gathered}
$$

and it suffices to assume $\nu=\gamma^{2}>3 \lambda^{2}$, in order to assure $\lambda$ to be the principal eigenvalue of $* d$ on $\mathcal{H}_{S^{1}}$.

We may think about $\lambda \neq 0$ as a "topological deviation" from the $\lambda=0$ case. We note that Hopf fields are principal curl eigenfields of $* d$ on $\mathcal{H}$ and therefore, by Proposition 3.1, energy minimizers. Lemma 4.2 and 3.2, provide a methodology to construct energy minimizers on products: $S^{1} \times \Sigma$. In the case of a $(K, \lambda)$ - structure, however, $\eta$ seem to be the most natural candidate for the energy minimizer on $\Xi_{\eta}$. The following special case, shows when $\eta$ becomes the principal curl eigenform on $\mathcal{H}$.

We choose a special bundle metric $\hat{g}_{P}$ on a principle circle bundle $P$, of constant length $l, S^{1}$ - fibers. Let $X$ a unit Killing vector field, tangent to the fibers such that $\eta=X^{b}$ defines a $(K, \lambda)$ - structure on $P$. In addition we assume that there always exists an $X$-invariant local frame of vector fields $\left\{X_{1}, X_{2}, X_{3}\right\}, X_{1}=X$, on $P$, namely

$$
\left[X_{1}, X_{i}\right]=0, \quad i=1,2,3 .
$$

Under this assumption and (22), (23) we obtain

$$
\mathcal{L}_{X} \eta_{i}=0, \quad \eta_{i}=X_{i}^{b} .
$$

Theorem 5.3. The curl eigenform $\eta$ defined by a $(K, \lambda)$-structure on $\left(P, \hat{g}_{P}\right)$ is a principal curl eigenform on $\mathcal{H}$, if

$$
\lambda^{2}<\min \left(\frac{\nu}{3}, \frac{4 \pi^{2}}{l^{2}}\right),
$$

where $\nu$ is the first nonzero eigenvalue of the scalar Laplacian $\Delta_{\Sigma}^{0}$ on $\Sigma$, and $l$ the length of the fibre.

Proof. Observe that on $\left(P, \hat{g}_{P}\right)$ the operator $-\mathcal{L}_{X}^{2}$ commutes with $\Delta_{P}^{1}=$ $\delta d+d \delta$. We check that $\mathcal{L}_{X}: \Omega^{*}(P) \rightarrow \Omega^{*}(P)$ commutes with the Hodge star operator $*: \Omega^{*}(P) \rightarrow \Omega^{*}(P)$. Indeed since $\mathcal{L}_{X}$ respects the wedge product:

$$
\mathcal{L}_{X}\left(\omega_{1} \wedge \omega_{2}\right)=\mathcal{L}_{X} \omega_{1} \wedge \omega_{2}+\omega_{1} \wedge \mathcal{L}_{X} \omega_{2}, \quad \omega_{1} \in \Omega^{j}(P), \omega_{2} \in \Omega^{k}(P),
$$

and the property (31), for any $k$ - form $\alpha=\sum_{I} a_{I} \omega_{I}$, where $\omega_{I}=\eta_{i_{1}} \wedge \eta_{i_{2}} \wedge$ $\ldots \wedge \eta_{i_{k}}, I=\left(i_{1}, \ldots, i_{k}\right)$ we have

$$
\mathcal{L}_{X} \alpha=\sum_{I}\left(\mathcal{L}_{X} a_{I}\right) \omega_{I}, \quad \text { since } \mathcal{L}_{X} \omega_{I}=0
$$


Consequently, $* \mathcal{L}_{X}=\mathcal{L}_{X} *$ follows from the definition of the Hodge star operator,

$$
* \mathcal{L}_{X} \alpha=\sum_{I}\left(\mathcal{L}_{X} a_{I}\right) * \omega_{I}=\mathcal{L}_{X} \sum_{I} a_{I} * \omega_{I}=\mathcal{L}_{X} * \alpha,
$$

since $\mathcal{L}_{X} \omega_{I}=\mathcal{L}_{X} * \omega_{I}=0$. Hence, we obtain $\mathcal{L}_{X} \Delta_{P}^{1}=\Delta_{P}^{1} \mathcal{L}_{X}$, because $\mathcal{L}_{X}$ commutes with an exterior derivative $d$ and $\delta= \pm * d *$. This in turn implies

$$
\left(-\mathcal{L}_{X}^{2}\right) \Delta_{P}^{1}=\Delta_{P}^{1}\left(-\mathcal{L}_{X}^{2}\right)
$$

Consequently, we may define the decomposition

$$
\Delta_{P}^{1}=-\mathcal{L}_{X}^{2}+\Delta_{P}^{H} .
$$

where $\Delta_{P}^{H}=\Delta_{P}^{1}+\mathcal{L}_{X}^{2}$, and we call $\Delta_{P}^{H}$ a horizontal Laplacian. Both $-\mathcal{L}_{X}^{2}$ and $\Delta_{P}^{H}$ are not elliptic since take into account only derivatives in certain directions, but $-\mathcal{L}_{X}^{2}$, and $\Delta_{P}^{H}$, have discrete spectra, and commute by (34). Consequently, these operators are simultaneously diagonalizable in a suitable $L^{2}$ - orhonormal basis of $\Delta_{P}^{1}$ - eigenforms (see also [3]). The decomposition (35) and (34) imply that any eigenvalue $\tau$ of $\Delta_{P}^{1}$ is a sum $\tau=\psi+\mu$ of eigenvalue: $\psi$ of $-\mathcal{L}_{X}^{2}$, and $\mu$ of $\Delta_{P}^{H}$.

If $-\mathcal{L}_{X}^{2} \alpha=\psi \alpha, \alpha=a_{i} \eta_{i}$, then

$$
-\mathcal{L}_{X}^{2} a_{i}=\psi a_{i}
$$

Solving this equation in a local chart $(t, q) \in U \simeq S^{1} \times V \subset P, V \subset \Sigma$, gives us $-\mathcal{L}_{X}^{2} a_{i}=-\partial_{t}^{2} a_{i}(t, q)=\psi a_{i}(t, q)$, for a fixed $q: a_{i}(t, q)=A_{q} \cos (\psi t)+$ $B_{q} \sin (\psi t)$. Therefore $\psi=(2 \pi n / l)^{2}, n \in \mathbb{N}$. Now the theorem is a consequence of: $\mathcal{H}_{S^{1}}=\operatorname{Ker}\left(-\mathcal{L}_{X}^{2}\right) \cap \mathcal{H}$, and Proposition 5.2.

The $(K, \lambda)$-structures of Theorem 5.3 , (i.e. satisfying the assumption (30)) are completely described in [13]. Their construction is based on the BoothbyWang construction (see also [4]). One builds the metric $\hat{g}_{P}$ on $P$ as follows: choose a Riemannian metric $g_{\Sigma}$ on $\Sigma$ and a connection $H_{\omega}$ on $P$ such that the connection 1-form $\omega$ satisfies,

$$
d \omega=-\Omega=-2 \mathcal{E} \sigma, \quad \sigma=\pi_{*}\left(*_{\Sigma} 1\right), \quad \pi: P \rightarrow \Sigma,
$$

where $\mathcal{E}$ is a degree of the bundle $P$, and $\Omega$ the curvature form of the connection. We define $\eta=\omega / \gamma, \gamma=$ const, and $X$ to be the unique vertical vector field such that $\eta(X)=1$. Now we define $\hat{g}_{P}$ as follows,

$$
\hat{g}_{P}(X, X)=1, \quad \hat{g}_{P}(V, W)=g_{\Sigma}\left(\pi^{*} V, \pi^{*} W\right), \quad \text { if } V, W \text { horizontal. }
$$

Clearly $\mathcal{L}_{X} \hat{g}_{P}=0$, therefore $X$ is a Killing vector field, and by (36),

$$
d \omega=\gamma d \eta=-2 \mathcal{E} \sigma=-2 \mathcal{E} * \eta,
$$

which results in $* d \eta=(-2 \mathcal{E} / \gamma) \eta$, and a $(K,-\mathcal{E} / \gamma)$ structure: $\left(P, \eta, \hat{g}_{P}\right)$. By this construction $\hat{g}_{P}$ also satisfies (30). 
Conjecture 5.4. The curl eigenform $\eta$ defined by a $(K, \lambda)$-structure is always a tight energy-minimizer on $\Xi_{\eta}$.

\section{ACKNOWLEDGMENTS}

We would like to thank the second referee for insightful comments, especially for Remark 4.5. The second author is grateful to Jason Cantarella for the inspiring discussion during Georgia Topology Conference in Athens 2000.

\section{REFERENCES}

[1] V. Arnold and B. Khesin. Topological Methods in Hydrodynamics, volume 125 of Applied Mathematical Sciences. Springer-Verlag, New York, 1998.

[2] D. Bennequin. Entrelacements et équations de Pfaff. Astérisque, 87-161, Soc. Math. France, Paris, 1983.

[3] L. Bergery and J. Bourguignon. Laplacians and Riemannian submescions with totally geodesic fiberes. Illinois J. of Math., 26(2):181-199, 1982.

[4] D. Blair. Riemannian geometry of contact and symplectic manifolds, volume 203 of Progress in Mathematics. Birkhäuser Boston Inc., Boston, MA, 2002.

[5] S. Chandrasekhar and P. Kendall. On force-free magnetic fields. Astrophys. J., 126:457460, 1957.

[6] C. Chicone. The topology of stationary curl parallel solutions of Euler's equations. Israel J. Math. 39:1-2, 161-166, 1981.

[7] S. S. Chern and R. S. Hamilton. On Riemannian metrics adapted to three-dimensional contact manifolds. In Workshop Bonn 1984 (Bonn, 1984), volume 1111 of Lecture Notes in Math., pages 279-308. Springer, Berlin, 1985.

[8] Y. Eliashberg. Classification of overtwisted contact structures on 3-manifolds. Invent. Math. 98:3, 623-637, 1989.

[9] J. Etnyre and R. Ghrist. Contact topology and hydrodynamics. I. Beltrami fields and the Seifert conjecture. Nonlinearity, 13(2):441-458, 2000.

[10] E. Giroux. Structures de contact sur les variétés fibrées en cercles audessus d'une surface. Comment. Math. Helv., 76(2):218-262, 2001.

[11] J. Jost. Riemannian geometry and geometric analysis. Universitext, Berlin: SpringerVerlag, third ed., 2002.

[12] R. Komendarczyk. On the contact geometry of nodal sets, math.dg/0402070. To appear, Trans. of Amer. Math. Soc., 2004.

[13] L. Nicolaescu. Adiabatic limits of the Seiberg-Witten equations on Seifert manifolds. Comm. Anal. Geom., 6(2):331-392, 1998.

[14] K. Uhlenbeck. Generic properties of eigenfunctions. Amer. J. Math., 98(4):1059-1078, 1976.

[15] Z. Yoshida and Y. Giga. Remarks on spectra of operator rot. Math. Z., 204(2):235-245, 1990.

Department of MATHEMATICS AND COORdinAted SCIENCES LABORATORY, UniverSITY OF ILLINOIS, URBANA IL, 61801

Department of Mathematics, Georgia Institute of Technology, Atlanta GA, 30332-0160 